\title{
Monitoring of Anxiety-Like Behaviors on Rats with Video Tracking Technology
}

\author{
Ayşen ÇETİN KARDEŞLER*1, Meriç ÇETİN ${ }^{2}$, Selami BEYHAN ${ }^{3}$
}

Accepted $3^{\text {rd }}$ September 2016

\begin{abstract}
Artificial sweeteners like MSG (Mono Sodium Glutamate) model has been used anxiety-like behaviors on rats. The tracking of rat's movements has broad applicability to questions in anxiety-like behaviors with different doses MSG injections $(50 \mathrm{mg} / \mathrm{kg} / \mathrm{day}, 100$ $\mathrm{mg} / \mathrm{kg} / \mathrm{day}$ and $200 \mathrm{mg} / \mathrm{kg} / \mathrm{day}$ ) to rats. In this paper, in order to measure three types locomotor activity (line crossing, rearing, grooming), a video tracking software is used. The advantage of this type of tracking software is that it provides to give locomotor activity of rats in real-time. The experimental results obtained in this study have shown that learning and memorial functions negatively affected in the brains of the rats an anxiety-like model. In addition, the visual tracking results demonstrate that video tracking system provides an accurate monitoring of rat's behavior.
\end{abstract}

Keywords: Rat, locomotor activity, anxiety-like behaviors, MSG, computerized video tracking, animal tracking.

\section{Introduction}

The etiology of the behavioral disorders might be genetic, physical, developmental and also psychological or social as well. Neurological disorder appears mostly with anxiety disorder and depression. In animals, behaviors likely to anxiety can be identified through experimental open field test, light/dark test, elevated plus maze test, tail suspension test, forced swim test, social interaction task [1]. Open field test enables the evaluation of parameters such as line crossing, rearing, grooming and the defecation number in rats [2]. Studies in rodents have shown that the administration of MSG at the neonatal stage alterations in locomotor activity have been shown [3-4]. Grooming duration and rearing counts can be used to assess anxiety-like and exploratory behaviors in rodents [5-7]. Following acute MSG administration, locomotor activity reduced, grooming increased, while rearing activity reduced at MSG doses [8]. Reduced locomotor activity can be considered a form of anxiety-like behavior [9].

The video tracking systems in open field tests (including mazes) has become a very popular technique for monitoring of anxietylike behaviors on rats. The automated observation systems, which always work in the same way therefore behaviors are recorded more reliably, provide to researchers many behavioural information about animals. In literature, various types of the techniques can be found, including infrared photobeam detectors [10-11-12-13], vibration detection [14], radio telemetry [15], radar [16] and computerized video analysis [17], [18]. However, video based tracking systems using video cameras are currently the most common approach to achieve the task [19]. As videocamera technology has preferred to obtain the desired outputs,

\footnotetext{
${ }^{1}$ Pamukkale University, Medicine Faculty, Department of Medical

Biochemistry Kınıkl, 20040, Denizli, Turkey

${ }^{2}$ Pamukkale University, Computer Engineering Department, , Kınıklı, 20040, Denizli, Turkey

${ }^{3}$ Pamukkale University, Electrical and Electronics Engineering Department, Kınıkl, 20040, Denizli, Turkey

*Corresponding Author: Email: aysencetin@yahoo.com

Note: This paper has been presented at the $3^{\text {rd }}$ International Conference on Advanced Technology \& Sciences (ICAT'16) held in Konya (Turkey), September 01-03, 2016.
}

many automated video tracking methods have been developed [20]. In those methods, analog video signals are sent to video processing unit which detects peaks of the signal. The detected signals indicate contrast between the tracked animal and the background. Then the coordinates of the tracked animal are determined. Modern video tracking systems generally digitize analog video signals using frame grabbers. The digitized video frames is composed of pixels and each pixel has gray scale value [21]. The tracking procedure analyzes digitized frame in order to pick out the animal from the background. Then, the desired calculations are performed in order to explain the meaning of animal's behaviour.

In this study, the anxiety-like behaviors are investigated using pharmacological activation model with MSG. The objective of this study is to evaluate the effects of neonatal exposure to MSG on locomotor activity with video tracking system.

This paper is organized as follows: In Section 2, material and method, which are designed here, are explained in detail. Section 3 presents the numerical results of the monitoring behaviors on rats with video tracking technique. The discussion and conclusions about the observation results are given in Section 4.

\section{Material and Method}

In this study, in order to measure various locomotor activity (such as line crossing, rearing and grooming) an automated video tracking mechanism is designed.

\subsection{Testing Procedure}

The open field test setup consists of a square field with a dimension of $45 \times 45 \mathrm{~cm}$ and it is lightened by 3 fluorescent bulbs. The top of the field is open to the ambient and the square field is bordered by white stripes each of $1 \mathrm{~cm}$ width, on a black colored background. Each of the experimental animals are brought to the center of the open field setup and released to move freely. Duration of the test is determined as 3 minutes during which the rats are observed closely and any of their behaviors are recorded as line crossing, rearing and grooming. Figure 1 shows the open field test area and the used video tracking mechanism is illustrated in Figure 2. 


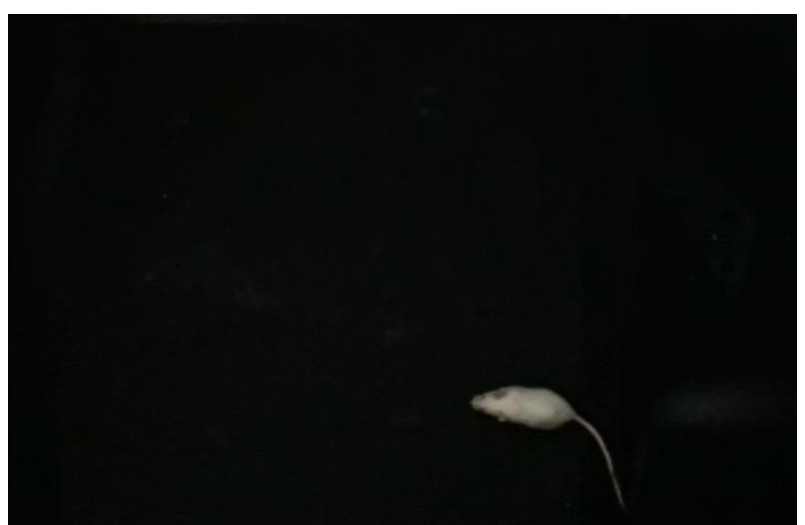

Figure 1. Open field test area.

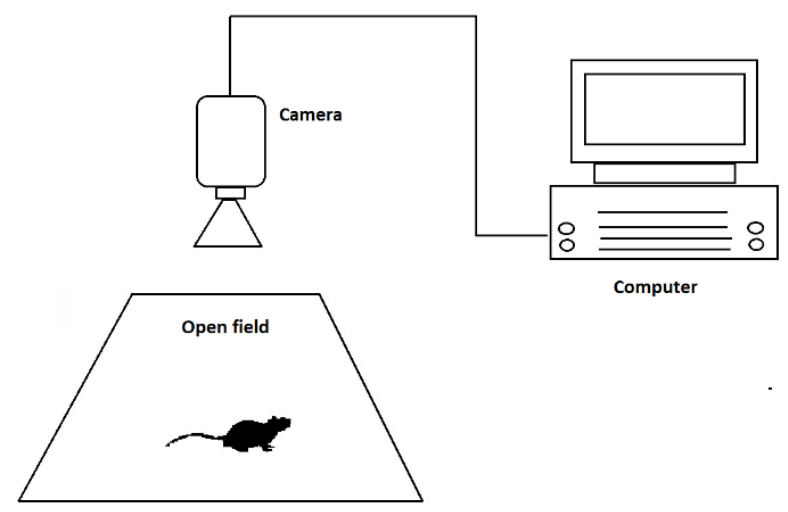

Figure 2. Video tracking mechanism of the open field test.

The video camera records the open field setup from the top where the rats are free in this area. Then, the camera signals synchronously are sent to a PC. The signals are digitized by frame grabbers. The video processing software analyses each frame in order to distinguish the tracked objects from the background on the basis of their gray scale since the gray scaling is the fastest. The threshold values of the video signals, which are used to subtract rat's image from the background, are set manually. Then, the coordinates of the animal are determined and recorded. Then, the monitoring of animal behavior is achieved in the open field maze.

\subsection{Experimental Animal Group}

Rats that are included in the study, are subject to 8 sessions of MSG injections in total, in the order of $50 \mathrm{mg} / \mathrm{kg} / \mathrm{day}$ (group number =6), $100 \mathrm{mg} / \mathrm{kg} / \mathrm{day}$ (group number = 6) and 200 $\mathrm{mg} / \mathrm{kg} / \mathrm{day}$ (group number $=6$ ). Rats that are selected as the control group (group number $=6$ ), are subject to the intraperitoneal serum physiologic.

\subsection{Hardware and Software Equipment}

Video data were processed with a PC (Windows 10 Pro platform, Intel Core i7-4600U CPU @ 2.10GHz $2.70 \mathrm{GHz}$ processor). Video frames were obtained by Flaxes FC 1604 camera (30fbs/15fbs). The data processing algorithms were developed using MATLAB 2014b and Image Processing, Computer Vision toolboxes.

\subsection{Data Analysis}

To analyze the data, we used an analysis of variance (ANOVA) for multiple groups. More complex designs, such as one-way
ANOVA with repeated measures or n-way ANOVA (additional factors: time, treatment, genotype, stress, etc), can also be used on behavioral studies of rats.

\section{Observation Results}

In this section, the observed rat's movements are illustrated separately in certain moments as shown in Figure 3. In addition, comparative tables related to locomotor activity are shown to discuss the behaviors of rats.

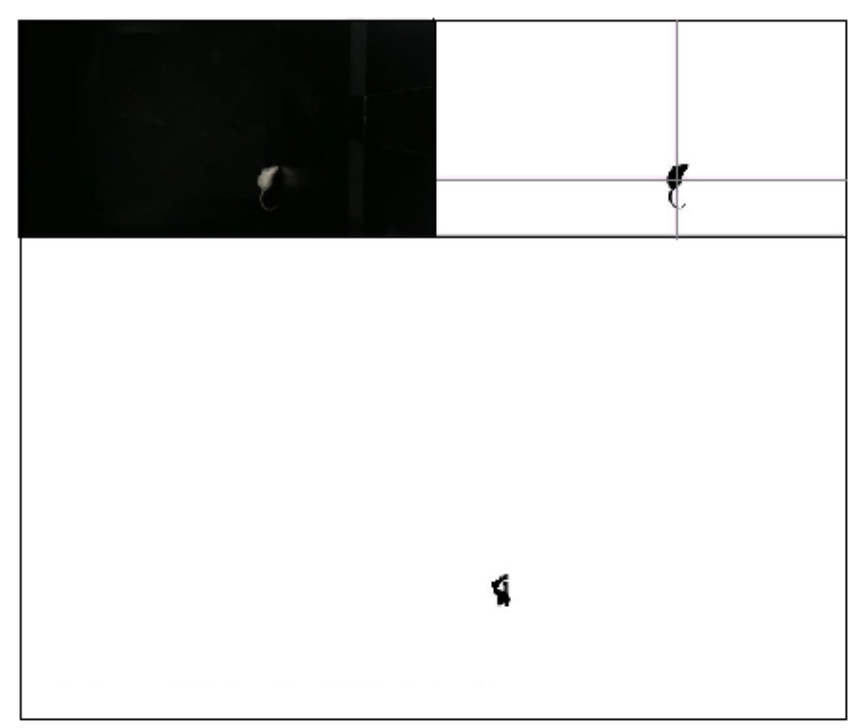

(a) 5th second

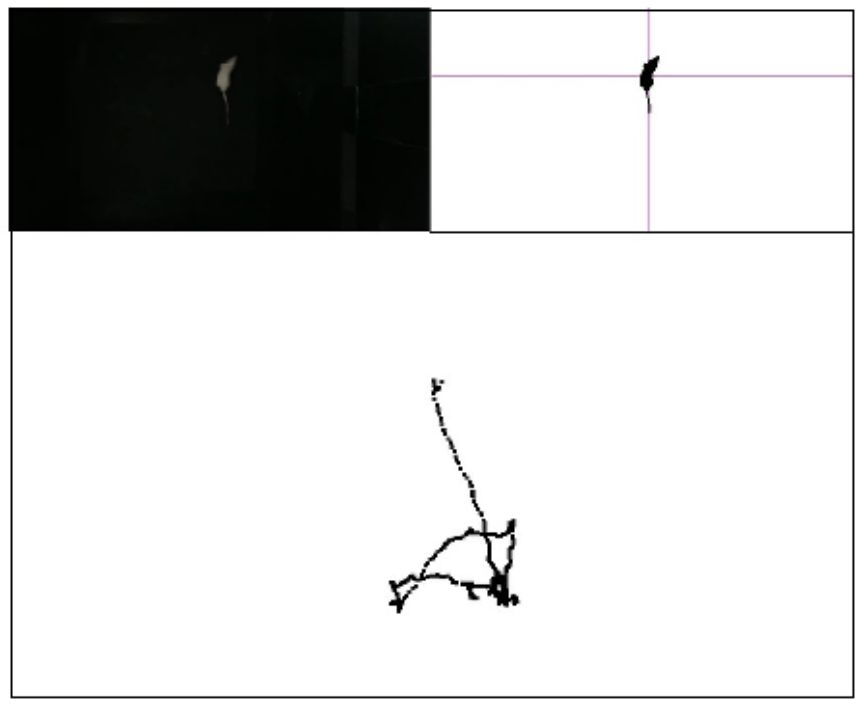

(b) 20th second 


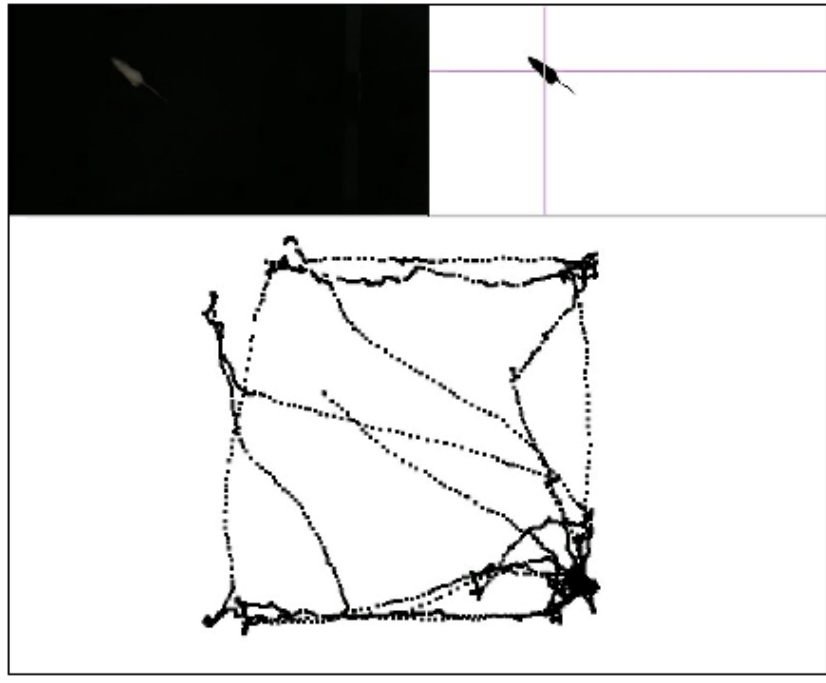

(c) 160th second

Figure 3. Observed rat's movements for several moments

Following the MSG injections, when the characteristics of anxiety resembling behaviors of the rats are monitored, a statistically considerable decrease is observed $(p<0.05)$ in the line crossing of the animals in the open field (Table 1).

Table 1. ANOVA values of line crossing and descriptive statistics, referred to the open field test values of the rats, pre and post injections of MSG

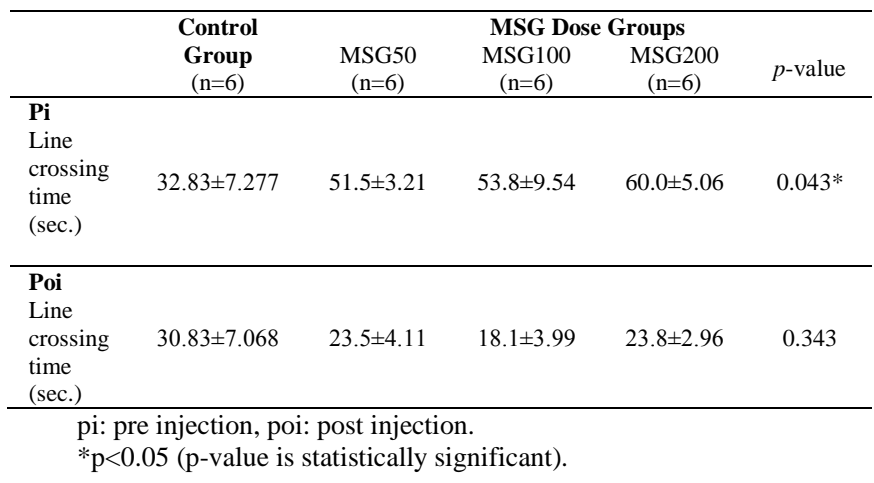

Descriptive statistics are point out Mean \pm standard error in Table 1. In addition to online tracking of the rat, designed video tracking software can automatically detect the other locomotor activities such as rearing and grooming. The numerical results related to rearing are presented in Table 2 .

Table 2. ANOVA values of rearing and descriptive statistics, referred to the open field test values of the rats, pre and post injections of MSG

\begin{tabular}{ccccc}
\hline $\begin{array}{c}\text { Control } \\
\text { Group } \\
(\mathrm{n}=6)\end{array}$ & MSG50 & MSG Dose Groups \\
$(\mathrm{n}=6)$ & $(\mathrm{n}=6)$ & $(\mathrm{n}=6)$ & $p$-value \\
\hline
\end{tabular}

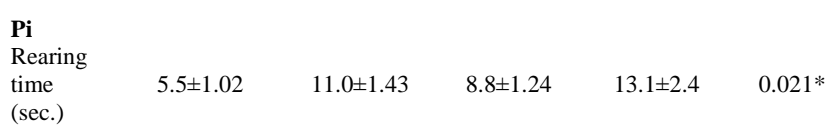

\begin{tabular}{lllll}
\hline $\begin{array}{l}\text { Poi } \\
\text { Rearing }\end{array}$ & $3.1 \pm 1.04 \quad 4.0 \pm 1.09$ & $2.6 \pm 0.4$ & $3.1 \pm 0.6$ & 0.740 \\
time & & & \\
(sec.) & & & \\
\hline pi: pre injection, poi: post injection. \\
${ }^{*}$ p $<0.05$ (p-value is statistically significant).
\end{tabular}

As shown in Table 2, following acute MSG administration rearing activity reduced at higher MSG doses. However, grooming values (Table 3 ) of the rats are increased on the contrary, by statistically reasonable figures $(\mathrm{p}<0.05)$.

Table 3. ANOVA values of line crossing and descriptive statistics, referred to the open field test values of the rats, pre and post injections of MSG

\begin{tabular}{|lccccc}
\hline & $\begin{array}{c}\text { Control } \\
\text { Group } \\
(\mathrm{n}=6)\end{array}$ & $\begin{array}{c}\text { MSG50 } \\
(\mathrm{n}=6)\end{array}$ & $\begin{array}{c}\text { MSG Dose Groups } \\
(\mathrm{n}=6)\end{array}$ & $\begin{array}{c}\text { MSG200 } \\
(\mathrm{n}=6)\end{array}$ & $p$-value \\
\hline $\begin{array}{l}\text { Pi } \\
\begin{array}{l}\text { Grooming } \\
\text { time (sec.) }\end{array}\end{array}$ & $16.5 \pm 1.78$ & $19.1 \pm 9.41$ & $26.0 \pm 12.11$ & $42.1 \pm 9.51$ & 0.218 \\
\hline $\begin{array}{l}\text { Poi } \\
\begin{array}{l}\text { Grooming } \\
\text { time (sec.) }\end{array}\end{array}$ & $14.0 \pm 1.78$ & $52.0 \pm 12.07$ & $49.6 \pm 9.82$ & $39.5 \pm 9.28$ & $0.030^{*}$ \\
\hline \multicolumn{2}{c}{ pi: pre injection, poi: post injection. } \\
$* \mathrm{p}<0.05$ (p-value is statistically significant).
\end{tabular}

Following post injection of MSG, grooming is increased significantly at doses of $50 \mathrm{mg} / \mathrm{kg} / \mathrm{day}$ and $100 \mathrm{mg} / \mathrm{kg} / \mathrm{day}$, respectively. It is known that the following acute MSG administration, locomotor activity reduced, grooming increased, while rearing activity reduced at MSG doses. Reduced locomotor activity is considered a form of anxiety-like behavior.

\section{Discussion and Conclusion}

In the literature, studies based on the anxiety model are achieved on animals through injections of artificial sweeteners such as MSG [22-23-24]. In the present study, characteristics of anxiety like behaviors of rats are determined through the injections of MSG at repetitive dosages. Following to the MSG injections, when we monitored the behavioral characteristics of rats resembling to anxiety, we determined a statistically considerable decrease in the roaming time of the animals in the open field and a decrease in the number of rearing behavior. However, the number of grooming is increased in a statistically considerable scale. Similar results is provided that a decrease in the motor activity is argued in [25-26].

As an overall result, we have observed that in the anxiety modelling of rats, over activation of astrocytes has a certain effect on the motor neuron functions of the animals. Additionally, we have observed that the neuronal development, neuronal survival and the synaptic plasticity are also negatively affected by the over activation of astrocytes. Computerized video-tracking results of rat's behavior confirm that the locomotor activities are negatively affected on rats an anxiety-like model.

\section{References}

[1] S. Vinogradov, et al. Is serum brain-derived neurotrophic factor a biomarker for cognitive enhancement in schizophrenia? Biol Psychiatry.; vol. 66, pp. 549-553, 2009.

[2] P. Ma, X . Liu, J . Wu, B . Yan, Y . Zhang, Y. Lu, Y. Wu, C . Liu, J. Guo, E. Nanberg, C.G. Bornehag, X. Yang. Cognitive deficits and anxiety induced by diisononyl phthalatein mice and the neuroprotective effects of melatonin, Nature. Vol. 5, pp. e14676, 2015.

[3] M. Dubovicky, D. Tokarev, I. Skultetyova, D. Jezova. Changes of exploratory behavior and its habituation in rats neonatally treated with monosodium glutamate, Pharmacol Biochem Behav, vol, 56, pp. 565-569, 1997.

[4] Z. Hlinak, D. Gandalovicova \& I. Krejci. Behavioral deficits in adult rats treated neonatally with glutamate, Neurotoxicol Teratol, vol. 27, pp. 465-473, 2005. 
[5] N. Enginar, I. Hatipoglu, ' M. Firtina, Evaluation of the acute effects of amitriptyline and fluoxetine on anxiety using grooming analysis algorithm in rats, Pharmacol. Biochem. Behav. Vol. 89, pp. 450-455, 2008.

[6] T.S. Perrot-Sinal, A. Gregus, D. Boudreau, L.E. Kalynchuk, Sex and repeated restraint stress interact to affect cat odorinduced defensive behavior in adult rats, Brain Res., vol. 1027, pp. 161-172, 2004.

[7] A. Takeda, H. Tamano, F. Kan, H. Itoh, N. Oku, Anxietylike behavior of young rats after 2-week zinc deprivation, Behav. Brain Res., vol. 177, pp. 1-6, 2007.

[8] O.J. Onaolapo, A.Y. Onaolapo, M.A. Akanmu, G. Olayiwola, Foraging enrichment modulates open field response to monosodium glutamate in mice. Annals of neurosciences, vol. 22.3, pp. 162, 2015.

[9] P. Muigg, S. Scheiber, P. Salchner, M. Bunck, R. Landgraf, N. Singewald, Differential stress-induced neuronal activation patterns in mouse lines selectively bred for high, normal or low anxiety, PLoS One vol. 4, e5346, 2009.

[10] RJ. Beninger, TA. Cooper, EJ. Mazurski, "Automating the measurement of locomotor activity", Neurobehav Toxicol Teratol, vol. 7, pp. 79-85, 1985.

[11] RL. Clarke, RF. Smith, DR. Justesen, "An infrared device for detecting locomotor activity", Behav Res Methods Instrum Comput, vol. 17, pp. 519-525, 1985.

[12] E. Robles, "A method to analyze the spatial distribution of behavior", Behav Res Methods Instrum Comput, vol. 22, pp. 540-549, 1990.

[13] MH. Teicher, SL. Andersen, P. Wallace, DA. Klein, J. Hostetter, Development of an affordable hi-resolution activity monitor system for laboratory animals, Pharmacol Biochem Behav, vol. 54, pp. 479-483, 1996.

[14] HA. Van de Weerd, RJ. Bulthuis, AF. Bergman, F. Schlingmann, J. Tolboom, PL. Van Loo et al., Validation of a new system for the automatic registration of behaviour in mice and rats. Behav Processes, vol. 53, pp. 11-20, 2001.

[15] AM. Deveney, A. Kjellstrom, T. Forsberg, DM. Jackson. A pharmacological validation of radiotelemetry in conscious, freely moving rats. J Pharmacol Toxicol Methods, vol. 40, pp. 71-79, 1998.

[16] V. Pasquali, P. Renzi, On the use of microwave radar devices in chronobiology studies: an application with Periplaneta Americana, Behav Res Methods, vol. 37, pp. 522-527, 2005.

[17] LP. Noldus, AJ. Spink, RA. Tegelenbosch. EthoVision: a versatile video tracking system for automation of behavioral experiments. Behav Res Methods Instrum Comput, vol. 33, pp. 398-414, 2001.

[18] CV. Vorhees, KD. Acuff-Smith, DR. Minck, RE. Butcher, A method for measuring locomotor behavior in rodents: contrast-sensitive computer-controlled video tracking activity assessment in rats. Neurotoxicol Teratol, vol. 14 pp. 43-49, 1992.

[19] Q. Xu, C. Cai, H. Zhou, H. Ren, A video tracking system for limb motion measurement in small animals. In Optoelectronics and Image Processing, International Conference, vol. 1, pp. 181-184, 2010.

[20] S. C. Fu, K. M. Chan, L. S. Chan, D. T. P Fong, P. Y. P Lui, The use of motion analysis to measure pain-related behaviour in a rat model of degenerative tendon injuries. Journal of neuroscience methods, vol. 179.2, pp. 309-318, 2009.

[21] JA. Endler, On the measurement and classification of colour in studies of animal colour patterns. Biol J Linn Soc, vol. 41, pp. 315-352, 1990.

[22] BA. McCool, AM. Chappell. Chronic intermittent ethanol inhalation increases ethanol self-administration in both C57BL/6J and DBA/2J mice. Alcohol., vol. 49, pp. 111120, 2015.

[23] CB. Quines, SG. Rosa, JT. Da Rocha, BM. Gai, CF. Bortolatto, MF. Duarte, CW. Nogueira. Monosodium glutamate, a food additive, induces depressive-like and anxiogenic-like behaviors in young rats. Life Sciences, vol. 107, pp. 27-31, 2016

[24] SG. Rosa, CB. Quines, EC. Stangherlin, CW. Nogueira. Diphenyl diselenide ameliorates monosodium glutamate induced anxiety-like behavior in rats by modulating hippocampal BDNF-Akt pathway and uptake of GABA and serotonin neurotransmitters. Physiology \& Behavior, vol. 155, pp. 1-8, 2016.

[25] J. Sun, C. Wan, P. Jia, AH. Fanous, KS. Kendler, BP. Riley, et al. Application of systems biology approach identifies and validates GRB2 as a risk gene for schizophrenia in the Irish Case Control Study of Schizophrenia (ICCSS) sample. Schizophrenia research., vol. 125, pp. 201-208, 2011.

[26] NH. Woo, HK. Teng, CJ. Siao, et al. Activation of p75NTR by proBDNF facilitates hippocampal long-term depression. Nat Neurosci., vol. 8, pp. 1069-1077, 2005. 